\title{
Automatic Extraction of Subcategorization Frames for Czech*
}

\author{
Anoop Sarkar \\ CIS Dept, Univ of Pennsylvania \\ 200 South 33 rd Street, \\ Philadelphia, PA 19104 USA \\ anoopelinc. cis. upenn . edu
}

\author{
Daniel Zeman \\ Ústav formální a aplikované lingvistiky \\ Univerzita Karlova \\ Praha, Czechia \\ zemandufal.mff.cuni.cz
}

\begin{abstract}
We present some novel machine learning techniques for the identification of subcategorization information for verbs in Czech. We compare three different statistical techniques applied to this problem. We show how the learning algorithm can be used to discover previously unknown subcategorization frames from the Czech Prague Dependency Treebank. The algorithm can then be used to label dependents of a verb in the Czech treebank as either arguments or adjuncts. Using our techniques, we are able to achieve $88 \%$ precision on unseen parsed text.
\end{abstract}

\section{Introduction}

The subcategorization of verbs is an essential issue in parsing, because it helps disambiguate the attachment of arguments and recover the correct predicate-argument relations by a parser. (Carroll and Minnen, 1998; Carroll and Rooth, 1998) give several reasons why subcategorization information is important for a natural language parser. Machinereadable dictionaries are not comprehensive enough to provide this lexical information (Manning, 1993; Briscoe and Carroll, 1997). Furthermore, such dictionaries are available only for very few languages. We need some general method for the automatic extraction of subcategorization information from text corpora.

Several techniques and results have been reported on learning subcategorization frames (SFS) from text corpora (Webster and Marcus, 1989; Brent, 1991; Brent, 1993; Brent, 1994; Ushioda et al., 1993; Manning, 1993; Ersan and Charniak, 1996; Briscoe and Carroll, 1997; Carroll and Minnen, 1998; Carroll and Rooth, 1998). All of this work

\footnotetext{
* This work was done during the second author's visit to the University of Pennsylvania. We would like to thank Prof. Aravind Joshi, David Chiang, Mark Dras and the anonymous reviewers for their comments. The first author's work is partially supported by NSF (irant SBR 8920230. Many tools used in this work are the results of project No. VS96151 of the Ministry of Eclucation of the Crech Republic. The data (PDT) is thanks to grant No. 405/96/K214 of the Grant Agency of the Czech Republic. Both grants were given to the Institute of Formal and Applied Linguistics. Faculty of Mathematics and Physics. Charles University, Prague.
}

deals with English. In this paper we report on techniques that automatically extract SFs for Czech, which is a free word-order language, where verb complements have visible case marking.

Apart from the choice of target language, this work also differs from previous work in other ways. Unlike all other previous work in this area, we do not assume that the set of SFs is known to us in advance. Also in contrast, we work with syntactically annotated data (the Prague Dependency Treebank, PDT (Hajič, 1998)) where the subcategorization information is not given; although this might be considered a simpler problem as compared to using raw text, we have discovered interesting problems that a user of a raw or tagged corpus is unlikely to face.

We first give a detailed description of the task of uncovering SFs and also point out those properties of Czech that have to be taken into account when searching for SFs. Then we discuss some differences from the other research efforts. We then present the three techniques that we use to learn SFs from the input data.

In the input data, many observed dependents of the verb are adjuncts. To treat this problem effectively, we describe a novel addition to the hypothesis testing technique that uses subset of observed frames to permit the learning algorithm to better distinguish arguments from adjuncts.

Using our techniques, we are able to achieve $88 \%$ precision in distinguishing arguments from adjuncts on unseen parsed text.

\section{Task Description}

In this section we describe precisely the proposed task. We also describe the input training material and the output produced by our algorithms.

\subsection{Identifying subcategorization frames}

In general, the problem of identifying subcategorization frames is to distinguish between arguments and adjuncts among the constituents modifying a

\footnotetext{
'One of the anonymous reviewers pointed out that (Basili and Vindigni. 1998) presents a corpus-driven acquisition of subcategorization frames for Italian.
} 


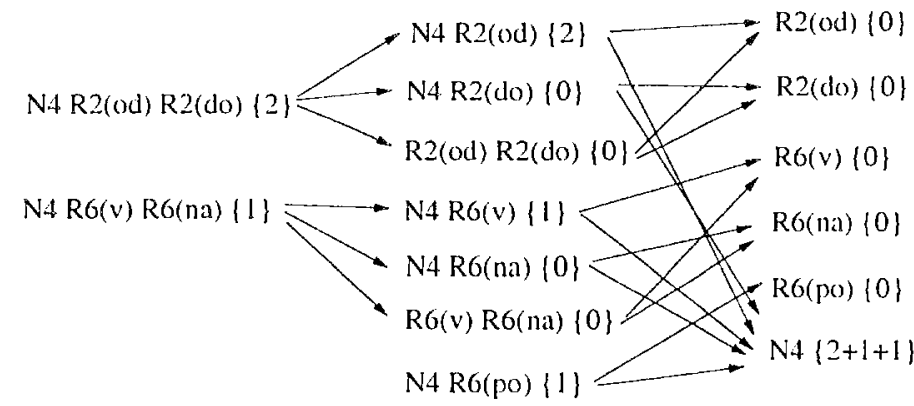

emply $\{0\}$

Figure 2: Computing the subsets of observed frames for the verb absolvovat. The counts for each frame are given within braces \{\} . In this example, the frames $N 4 R 2(o d)$. N4 R6 $(v)$ and $N 4 R 6(p o)$ have been observed with other verbs in the corpus. Note that the counts in this figure do not correspond to the real counts for the verb absolvovat in the training corpus.

where $c(\cdot)$ are counts in the training data. Using the values computed above:

$$
\begin{aligned}
p_{1} & =\frac{k_{1}}{n_{1}} \\
p_{2} & =\frac{k_{2}}{n_{2}} \\
p & =\frac{k_{1}+k_{2}}{n_{1}+n_{2}}
\end{aligned}
$$

Taking these probabilities to be binomially distributed, the log likelihood statistic (Dunning, 1993) is given by:

$$
\begin{aligned}
& -2 \log \lambda= \\
& 2\left[\log L\left(p_{1}, k_{1}, n_{1}\right)+\log L\left(p_{2}, k_{2}, n_{2}\right) \cdots\right. \\
& \left.\quad \log L\left(p, k_{1}, n_{2}\right)-\log L\left(p, k_{2}, n_{2}\right)\right]
\end{aligned}
$$

where,

$$
\log L(p, n, k)=k \log p+(n-k) \log (1-p)
$$

According to this statistic, the greater the value of $-2 \log \lambda$ for a particular pair of observed frame and verb, the more likely that frame is to be valid SF of the verb.

\subsection{T-scores}

Another statistic that has been used for hypothesis testing is the $t$-score. Using the definitions from Section 3.1 we can compute $t$-scores using the equation below and use its value to measure the association between a verb and a frame observed with it.

$$
T=\frac{p_{1}-p_{2}}{\sqrt{\sigma^{2}\left(n_{1}, p_{1}\right)+\sigma^{2}\left(n_{2}, p_{2}\right)}}
$$

where,

$$
\sigma(n, p)=n p(1-p)
$$

In particular, the hypothesis being tested using the t-score is whether the distributions $p_{1}$ and $p_{2}$ are not independent. If the value of $T$ is greater than some threshold then the verb $v$ should take the frame $f$ as a SF.

\subsection{Binomial Models of Miscue Probabilities}

Once again assuming that the data is binomially distributed, we can look for frames that co-occur with a verb by exploiting the miscue probability: the probability of a frame co-occuring with a verb when it is not a valid SF. This is the method used by several earlier papers on SF extraction starting with (Brent, 1991; Brent, 1993; Brent, 1994).

Let us consider probability $p_{\text {!f }}$ which is the probability that a given verb is observed with a frame but this frame is not a valid SF for this verb. $p_{\text {! }}$ is the error probability on identifying a $S F$ for a verb. Let us consider a verb $v$ which does not have as one of its valid SFs the frame $f$. How likely is it that $v$ will be seen $m$ or more times in the training data with frame $f$ ? If $v$ has been seen a total of $n$ times in the data, then $H^{*}\left(p_{!} ; m, n\right)$ gives us this likelihood.

$$
H^{*}\left(p_{! f} ; m, n\right)=\sum_{i=m}^{n} p_{! f}^{2}\left(1-p_{! f}\right)^{n-i}\left(\begin{array}{c}
n \\
i
\end{array}\right)
$$

If $H^{*}(p ; m, n)$ is less than or equal to some small threshold value then it is extremely unlikely that the hypothesis is true, and hence the frame $f$ must be a SF of the verb $v$. Setting the threshold value to 0.05 gives us a $95 \%$ or better confidence value that the verb $v$ has been observed often enough with a frame $f$ for it to be a valid SF.

Initially, we consider only the observed frames (OFs) from the treebank. There is a chance that some are subsets of some others but now we count only the cases when the OFs were seen themselves. Let's assume the test statistic rejected the frame. Then it is not a real SF but there probably is a subset of it that is a real SF. So we select exactly one of 
the subsets whose length is one member less: this is the successor of the rejected frame and inherits its frequency. Of course one frame may be successor of several longer frames and it can have its own count as OF. This is how frequencies accumulate and frames become more likely to survive. The example shown in Figure 2 illustrates how the subsets and successors are selected.

An important point is the selection of the successor. We have to select only one of the $n$ possible successors of a frame of length $n$, otherwise we would break the total frequency of the verb. Suppose there is $m$ rejected frames of length $n$. This yiclds $m * n$ possible modifications to consider before selection of the successor. We implemented two methods for choosing a single successor frame:

1. Choose the one that results in the strongest preference for some frame (that is, the successor frame results in the lowest entropy across the corpus). This measure is sensitive to the frequency of this frame in the rest of corpus.

2. Random selection of the successor frame from the alternatives.

Random selection resulted in better precision ( $88 \%$ instead of $86 \%$ ). It is not clear why a method that is sensitive to the frequency of each proposed successor frame does not perform better than random selection.

The technique described here may sometimes result in subset of a correct SF, discarding one or more of its members. Such frame can still help parsers because they can at least look for the dependents that have survived.

\section{Evaluation}

For the evaluation of the methods described above we used the Prague Dependency Treebank (PIT). We used 19,126 sentences of training data from the PD'T (about $300 \mathrm{~K}$ words). In this training set, there were 33,641 verb tokens with 2,993 verb types. There were a total of 28,765 observed frames (see Section 2.1 for explanation of these terms). There were 914 verb types seen 5 or more times.

Since there is no electronic valence dictionary for Czech, we evaluated our filtering technique on a set of 500 test sentences which were unseen and separate from the training data. These test sentences were used as a gold standard by distinguishing the arguments and adjuncts manually. We then compared the accuracy of our output set of items marked as either arguments or adjuncts against this gold standard.

First we describe the baseline methods. Baseline method 1: consider each dependent of a verb an adjunct. Baseline method 2: use just the longest known observed frame matching the test pattern. If no matching $\mathrm{OF}$ is known, find the longest partial match in the OFs seen in the training data. We exploit the functional and morphological tags while matching. No statistical filtering is applied in either baseline method.

A comparison between all three methods that were proposed in this paper is shown in Table 1.

The experiments showed that the method improved precision of this distinction from $57 \%$ to $88 \%$. We were able to classify as many as 914 verbs which is a number outperformed only by Manning, with $10 \mathrm{x}$ more data (note that our results are for a different language).

Also, our method discovered 137 subcategorization frames from the data. The known upper bound of frames that the algorithm could have found (the total number of the observed frame types) was 450 .

\section{Comparison with related work}

Preliminary work on SF extraction from corpora was done by (Brent, 1991; Brent, 1993; Brent, 1994) and (Webster and Marcus, 1989; Ushioda et al., 1993). Brent (Brent, 1993; Brent, 1994) uses the standard method of testing miscue probabilities for filtering frames observed with a verb. (Brent, 1994) presents a method for estimating $\eta_{!} f$. Brent applied his method to a small number of verbs and associated SF types. (Manning, 1993) applies Brent's method to parsed data and obtains a subcategorization dictionary for a larger set of verbs. (Briscoe and Carroll, 1997; Carroll and Minnen, 1998) differs from earlier work in that a substantially larger set of SF types are considered; (Carroll and Rooth, 1998) use an EM algorithm to learn subcategorization as a result of learning rule probabilities, and, in turn, to improve parsing accuracy by applying the verb SFs obtained. (Basili and Vindigni, 1998) use a conceptual clustering algorithm for acquiring subcategorization frames for Italian. They establish a partial order on partially overlapping OFs (similar to our OF subsets) which is then used to suggest a potential SF. A complete comparison of all the previous approaches with the current work is given in Table 2.

While these approaches differ in size and quality of training data, number of SF types (e.g. intransitive verbs, transitive verbs) and number of verbs processed, there are properties that all have in common. They all assume that they know the set of possible SF types in advance. Their task can be viewed as assigning one or more of the (known) SF types to a given verb. In addition, except for (Briscoe and Carroll, 1997; Carroll and Minnen, 1998), only a small number of SF types is considered. 


\begin{tabular}{|l|c|c|c|c|c|}
\hline & Baseline 1 & Baseline 2 & Lik. Ratio & T-scores & Hyp. Testing \\
\hline Precision & $55 \%$ & $78 \%$ & $82 \%$ & $82 \%$ & $88 \%$ \\
Recall: & $55 \%$ & $73 \%$ & $77 \%$ & $77 \%$ & $74 \%$ \\
$F_{\beta=1}$ & $55 \%$ & $75 \%$ & $79 \%$ & $79 \%$ & $80 \%$ \\
$\%$ unknown & $0 \%$ & $6 \%$ & $6 \%$ & $6 \%$ & $16 \%$ \\
\hline Total verb nodes & 1027 & 1027 & 1027 & 1027 & 1027 \\
Total complements & 2144 & 2144 & 2144 & 2144 & 2144 \\
Nodes with known verbs & 1027 & 981 & 981 & 981 & 907 \\
Complements of known verbs & 2144 & 2010 & 2010 & 2010 & 1812 \\
Correct Suggestions & 1187.5 & 1573.5 & 1642.5 & 1652.9 & 1596.5 \\
True Arguments & 956.5 & 910.5 & 910.5 & 910.5 & 834.5 \\
Suggested Arguments & 0 & 1122 & 974 & 1026 & 674 \\
Incorrect arg suggestions & 0 & 324 & 215.5 & 236.3 & 27.5 \\
Incorrect adj suggestions & 956.5 & 112.5 & 152 & 120.8 & 188 \\
\hline
\end{tabular}

Table 1: Comparison between the baseline methods and the three methods proposed in this paper. Some of the values are not integers since for some difficult cases in the test data, the value for each argument/adjunct decision was set to a value between $[0,1]$. Recall is computed as the number of known verb complements divided by the total number of complements. Precision is computed as the number of correct suggestions divided by the number of known verb complements. $F_{\beta=1}=(2 \times p \times r) /(p+r)$. \% unknown represents the percent of test data not considered by a particular method.

Using a dependency treebank as input to our learning algorithm has both advantages and drawbacks. There are two main advantages of using a treebank:

- Access to more accurate data. Data is less noisy when compared with tagged or parsed input data. We can expect correct identification of verbs and their dependents.

- We can explore techniques (as we have done in this paper) that try and learn the set of SFs from the data itself, unlike other approaches where the set of SFs have to be set in advance.

Also, by using a treebank we can use verbs in different contexts which are problematic for previous approaches, e.g. we can use verbs that appear in relative clauses. However, there are two main drawbacks:

- Treebanks are expensive to build and so the techniques presented here have to work with less data.

- All the dependents of each verb are visible to the learning algorithm. This is contrasted with previous techniques that rely on finite-state extraction rules which ignore many dependents of the verb. Thus our technique has to deal with a different kind of data as compared to previous approaches.

We tackle the second problem by using the method of observed frame subsets described in Section 3.3 .

\section{Conclusion}

We are currently incorporating the SF information produced by the methods described in this paper into a parser for Czech. We hope to duplicate the increase in performance shown by treebank-based parsers for English when they use SF information. Our methods can also be applied to improve the annotations in the original treebank that we use as training data. The automatic addition of subcategorization to the treebank can be exploited to add predicate-argument information to the treebank.

Also, techniques for extracting SF information from data can be used along with other research which aims to discover relationships between different SFs of a verb (Stevenson and Merlo, 1999; Lapata and Brew, 1999; Lapata, 1999; Stevenson et al., 1999).

The statistical models in this paper were based on the assumption that given a verb, different SFs occur independently. This assumption is used to justify the use of the binomial. Future work perhaps should look towards removing this assumption by modeling the dependence between different SFs for the same verb using a multinomial distribution.

To summarize: we have presented techniques that can be used to learn subcategorization information for verbs. We exploit a dependency treebank to learn this information, and moreover we discover the final set of valid subcategorization frames from the training data. We achieve upto $88 \%$ precision on unseen data.

We have also tried our methods on data which was automatically morphologically tagged which 


\begin{tabular}{|c|c|c|c|c|c|c|}
\hline $\begin{array}{l}\text { Previous } \\
\text { work }\end{array}$ & Data & \#SFS & $\begin{array}{c}\text { \#verbs } \\
\text { tested }\end{array}$ & Method & $\begin{array}{l}\text { Miscue } \\
\text { rate }\end{array}$ & Corpus \\
\hline (Ushioda et al., 1993) & $\begin{array}{l}\text { POS + } \\
\text { FS rules }\end{array}$ & 6 & 33 & heuristics & $\mathrm{NA}$ & WSJ $(300 \mathrm{~K})$ \\
\hline (Brent, 1993) & $\begin{array}{l}\text { raw }+ \\
\text { FS rules }\end{array}$ & 6 & $1 \overline{9} 3$ & $\begin{array}{l}\text { Hypothesis } \\
\text { testing }\end{array}$ & $\begin{array}{l}\text { iterative } \\
\text { estimation }\end{array}$ & Brown $(1.1 \mathrm{M})$ \\
\hline (Manning, 1993) & $\begin{array}{l}\text { POS + } \\
\text { FS rules }\end{array}$ & 19 & 3104 & $\begin{array}{l}\text { Hypothesis } \\
\text { testing }\end{array}$ & hand & $\mathrm{NYT}(4.1 \mathrm{M})$ \\
\hline (Brent, 1994) & $\begin{array}{l}\text { raw }+ \\
\text { heuristics }\end{array}$ & 12 & 126 & $\begin{array}{l}\text { Hypothesis } \\
\text { testing }\end{array}$ & $\begin{array}{l}\text { non-iter } \\
\text { estimation }\end{array}$ & CHILDES (32K) \\
\hline (Ersan and Chamiak, 1996) & $\begin{array}{l}\text { Full } \\
\text { parsing }\end{array}$ & 16 & 30 & $\begin{array}{l}\text { Hypothesis } \\
\text { testing }\end{array}$ & hand & WSJ $(36 \mathrm{M})$ \\
\hline (Briscoe and Carroll, 1997) & $\begin{array}{l}\text { Full } \\
\text { parsing }\end{array}$ & 160 & 14 & $\begin{array}{l}\text { Hypothesis } \\
\text { testing }\end{array}$ & $\begin{array}{l}\text { Dictionary } \\
\text { estimation }\end{array}$ & various $(70 \mathrm{~K})$ \\
\hline (Carroll and Rooth, 1998) & Unlabeled & $9+$ & 3 & $\begin{array}{l}\text { Inside- } \\
\text { outside }\end{array}$ & NA & $\mathrm{BNC}(5-30 \mathrm{M})$ \\
\hline Current Work & $\begin{array}{l}\text { Fully } \\
\text { Parsed }\end{array}$ & $\begin{array}{c}\text { Learned } \\
137\end{array}$ & 914 & $\begin{array}{l}\text { Subsetst } \\
\text { Hyp. testing }\end{array}$ & Estimate & PDT $(300 \mathrm{~K})$ \\
\hline
\end{tabular}

Table 2: Comparison with previous work on automatic SF extraction from corpora

allowed us to use more data $(82 \mathrm{~K}$ sentences instead of $19 \mathrm{~K}$ ). The performance went up to $89 \%$ (a $1 \%$ improvement).

\section{References}

Roberto Basili and Michele Vindigni. 1998. Adapting a subcalegorization lexicon to a domain. In Proceedings of the ECML'98 Workshop TANI.PS: Towards adaptive NLP. driven systems: linguistic information, learning methods and applications, Chemunitz. Germany, Apr 24.

Peter Bickel and Kjell Doksum. 1977. Mathematical Statistics. Holden-Day Inc.

Michael Brent. 1991. Automatic acquistion of subcategorization frames from untagged text. In Proceedings of the $29 t h$ Me'ting of the ACL, pages 209-214. Berkeley, CA

Michael Brent. 1993. From grammar to lexicon: unsupervised learning of lexical syntax. Computational linguistics, 19(3):243-262.

Michael Brent. 1994. Acquisition of subcategorization frames using aggregated evidence from local syntactic cues. Lingua, 92:433-470. Reprinted in Acquisition of the Lexicon, L. Gleitman and B. Landau (Eds.). MIT Press, Cambridge, $\mathrm{MA}$

Ted Briscoe and John Carroll. 1997. Automatic extraction of subeategorization from corpora. In Procesdings of the 5 th ANLP Conference, pages 356-363, Washington. D.C. ACI.

John Carroll and Guido Minnen. 1998. Can subcategorisation probabilities help a statistical parser. In Proccedings of the Gth ACL/SIGDAT Workshop on Very large Corport (WVLC - 6 ), Montreal, Canadit.

Glenn Carroll and Mats Rooth. 1998. Valence induction with a head-lexicalized PCFG. In Proceedings of the 3 rd Conference on Empirical Methods in Natural Language Processing (EMNLP 3). Granadit. Spain.

Ted Dunning. 1993. Accurate methods for the statistics of surprise and coincidence. Computational Linguistics. 19(1):61-74, March.

Murat Ersan and Eugene Charniak. 1996. A statistical synlactic disambiguation program and what it learns. In S. Wermter, E. Riloff, and G. Scheler, editors, Connectionist, Statistical and Symbolic Approaches in Learning for Narural Language Processing. volume 1040 of Lechure Notes in Arrifical Intelligence, pages 146-159. SpringerVerlag, Berlin.

Jan Hajič and Barbora Hladká. 1998. Tagging intlective languages: Prediction of morphological categories for a rich, structured tagset. In Proceedings of COLING-ACL 98. Université de Montréal, Montréal, pages 483-490.

Jan Hajič. 1998. Building a syntactically annotated corpus: The prague dependency treebank. In Issues of Valency and Meaning, pages 106-132. Karolinum. Praha.

Maria Lapata and Chris Brew. 1999. Using subcategorization to resolve verb class ambiguity. In Pascale Fung and Joe Zhou, editors, Proceedings of WVLC\%MNIP, pages 260 274, 21-22 June.

Maria Lapata. 1999. Acquiring lexical generalizations from corpora: A case study for diathesis alternations. In Proceed ings of 37 th Meering of $A C L$, pages 397-404.

Christopher D. Manning. 1993. Automatic acquisition of a large subcategorization dictionary from corpora. In Proceedings of the 31st Meeting of the ACl, pages 235-242. Columbus, Ohio.

Suzame Stevenson and Paola Merlo. 1999. Automatic verb chassitication using distributions of grammatical features. In Procedings of EACL '99. pages 45-52, Bergen, Norway, 8-12 June.

Suzanne Stevenson, Paola Merlo, Natalia Kariacva, and Kamin Whitchouse. 1999. Supervised learning of lexical semantic classes using frequency distributions. In S/GLEX-99.

Akira Ushioda. David A. Evans, Ted Gibson, and Alex Waibel. 1993. The automatic acquisition of frequencies of verb subcatcgorization frames from tagged corpora. In B. Boguraev and J. Pustejovsky, editors, Proceedings of the Workshop on Acquisition of Lexical Knowledge from Teat, pages 95-106, Columbus, OH. 21 June.

Mort Webster and Mitchell Marcus. 1989. Automatic acquisition of the lexical frames of verbs from sentence frames. In Proceedings of the 27th Mecting of the ACL, pages 177-184 